\title{
Article \\ Accurate Receptor-Ligand Binding Free Energies from Fast QM Conformational Chemical Space Sampling
}

\author{
Esra Boz and Matthias Stein *(D)
}

check for updates

Citation: Boz, E.; Stein, M. Accurate Receptor-Ligand Binding Free Energies from Fast QM Conformational Chemical Space Sampling. Int. J. Mol. Sci. 2021, 22, 3078. https://doi.org/10.3390/ ijms22063078

Academic Editor: Alexander Baykov

Received: 15 February 2021

Accepted: 15 March 2021

Published: 17 March 2021

Publisher's Note: MDPI stays neutral with regard to jurisdictional claims in published maps and institutional affiliations.

Copyright: (c) 2021 by the authors. Licensee MDPI, Basel, Switzerland. This article is an open access article distributed under the terms and conditions of the Creative Commons Attribution (CC BY) license (https:/ / creativecommons.org/licenses/by/ $4.0 /)$.
Max Planck Institute for Dynamics of Complex Technical Systems, Molecular Simulations and Design Group, 39106 Magdeburg, Germany; boz@mpi-magdeburg.mpg.de

* Correspondence: matthias.stein@mpi-magdeburg.mpg.de

\begin{abstract}
Small molecule receptor-binding is dominated by weak, non-covalent interactions such as van-der-Waals hydrogen bonding or electrostatics. Calculating these non-covalent ligand-receptor interactions is a challenge to computational means in terms of accuracy and efficacy since the ligand may bind in a number of thermally accessible conformations. The conformational rotamer ensemble sampling tool (CREST) uses an iterative scheme to efficiently sample the conformational space and calculates energies using the semi-empirical 'Geometry, Frequency, Noncovalent, eXtended Tight Binding' (GFN2-xTB) method. This combined approach is applied to blind predictions of the modes and free energies of binding for a set of 10 drug molecule ligands to the cucurbit[n]urils $\mathrm{CB}[8]$ receptor from the recent 'Statistical Assessment of the Modeling of Proteins and Ligands' (SAMPL) challenge including morphine, hydromorphine, cocaine, fentanyl, and ketamine. For each system, the conformational space was sufficiently sampled for the free ligand and the ligand-receptor complexes using the quantum chemical Hamiltonian. A multitude of structures makes up the final conformer-rotamer ensemble, for which then free energies of binding are calculated. For those large and complex molecules, the results are in good agreement with experimental values with a mean error of $3 \mathrm{kcal} / \mathrm{mol}$. The GFN2-xTB energies of binding are validated by advanced density functional theory calculations and found to be in good agreement. The efficacy of the automated QM sampling workflow allows the extension towards other complex molecular interaction scenarios.
\end{abstract}

Keywords: ligand binding free energy; DFT; conformational sampling; ligand-receptor binding; GFN2-xTB; conformational entropy

\section{Introduction}

Binding free energy calculations on molecular systems are required to eventually predict binding affinities for (bio)molecular systems [1]. Especially during the last decade, the pharmaceutical industry and further research activities along this line are gaining continuous attention in order to obtain more efficient and selective drugs [2-4].

The use of computational drug design tools enable to reduce the traditional and time-consuming lab experiments in lead optimization [5]. These tools are also crucial to understand the underlying mechanisms of drug binding and metabolism. However, there are many factors in molecular recognition, which contribute to the accuracy of a calculation such as the sufficient sampling of the conformational space, energy evaluation schemes, maybe scoring functions for binding energy calculations, and the treatment of solvation. For example, enantioselective receptor binding of chiral compounds required a re-parametrization of atomic charges and torsional profiles, an exhaustive conformational Monte Carlo sampling plus a systematic low-mode conformational search followed by a normal mode integration approach to obtain enantiomeric excess in good agreement with experiments [6-8].

The number of computational approaches to calculate Gibbs free energies of binding is large and increasing: All-atom molecular dynamics (MD) simulation with explicit solvent 
molecules plus efficient free-energy sampling tools are, in principle, able to accurately predict binding free energies of ligands to proteins [9]. Free-energy simulation approaches are complex and include the double-decoupling method (DDM) and potential of mean force approach (PMF) augmented by either free-energy perturbation (FEP), thermodynamic integration (TI), or umbrella sampling. There are a number of comprehensive review articles, which address various aspects of free energy calculations [9-12].

In order to assess the performance of those approaches, Statistical Assessment of Modeling of Proteins and Ligands (SAMPL) blind prediction challenges were initiated to address various aspects of predictions of properties related to drug discovery [13-16]. Since 2008, those SAMPL competitions have addressed the accurate modeling of proteinligand complexes, but also small molecule properties such as hydration energies and the binding thermodynamics of host-guest systems. The most recent SAMPL8 was calling for predictions of 'drugs of abuse' binding to a synthetic receptor, including morphine, hydromorphine, methamphetamine, cocaine, and others. Cucurbit[n]urils, $\mathrm{CB}[\mathrm{n}]$, are macrocyclic synthetic receptors and constructed by glycoluril groups, which are connected by [n] units of methylene bridges [17]. Due to their high binding constants and non-toxic nature, they are very good candidates for biological applications [18]. CB[8] is the largest member of this family. It has a cavity diameter of $8.8 \AA$ and a cavity volume of $479 \AA^{3}$ (see Figure 1) [17]. These features make $C B[8]$ a very good candidate not only for $1: 1$, but also for possibly 1:2 host-guest complexation [19]. Binding constants $K_{a}$ in the range of $10^{4}-10^{12}$ $\mathrm{M}^{-1}$ of several $\mathrm{CB}[\mathrm{n}]$ complexes (with $\mathrm{CB}[6], \mathrm{CB}[7]$ and $\mathrm{CB}[8]$ as hosts) were measured by monitoring ${ }^{1} \mathrm{H}-\mathrm{NMR}$ spectra at neutral $\mathrm{pH}[20]$.
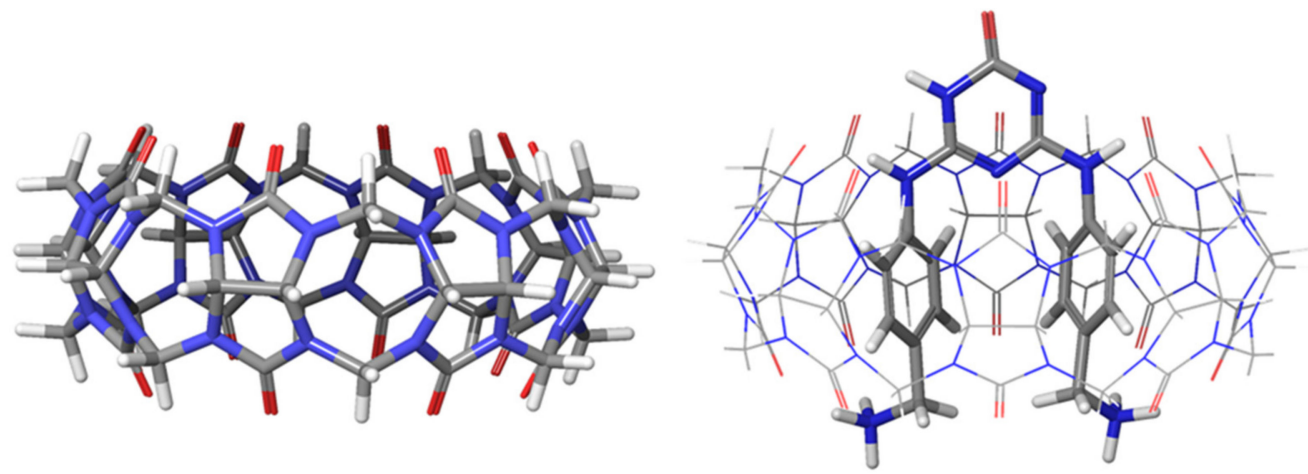

Figure 1. Structure of the host molecule CB[8] (side view) and co-crystallized with G0 ligand.

Cucurbit[n]urils were already included in previous challenges [14,16,21]. Several methods like MD simulations, meta-dynamic simulations, clustering, docking, umbrella sampling, $\mathrm{QM}$, and $\mathrm{QM} / \mathrm{MM}$ approaches were used to treat the given systems of the challenge [22-27]. Analysis of the submitted results revealed that the accuracy of force fields, inadequate consideration of different protonation states, and protomers of the structures were decisive factors for deviations from experiment [14].

Association free energies for supramolecular host-guest complexes can be computed with good accuracy by dispersion corrected density functional theory (DFT-D3) using a large basis set and one or a few manually generated possible binding modes [28,29].

Generally, for the modelling of proteins and biologically relevant large structures, classical MD simulations are preferred due to their low computational cost. These methods are usually lacking the required accuracy since even the most specialized force fields are missing some important parameters to describe the electronic properties of a system. Gibbs free energies of binding $\left(\Delta \mathrm{G}_{\mathrm{binding}}\right)$ are computationally demanding since exhaustive conformational sampling plus the accuracy of interaction energies are both essential.

Here, we present results of the automated exploration of the chemical space using CREST (Conformer-Rotamer Ensemble Sampling Tool), which makes use of the fast semiempirical QM tight-binding 'Geometry, Frequency, Noncovalent, eXtended Tight Binding' 
GFN2-xTB method [30]. GFN2-xTB is a new and extended semi-empirical tight-binding model, which is especially parametrized for geometries, frequencies, and non-covalent molecular interaction energies. A minimal valence basis set of atom-centered Gaussian functions is employed. Due to the inclusion of short-range dispersion interactions, the results are less empirical, and the method is more physically sound compared to other semi-empirical approaches. In contrast to previous semi-empirical methods, which rely on an element pair-specific parametrization, GFN2-xTB strictly follows a global and elementspecific parameter strategy in which no element pair-specific parameters are employed.

The CREST workflow makes use of iterative metadynamics plus a genetic Z-matrix crossing (iMTD-GC) approach to automatically sample the low-energy conformational space of ligands, receptors, and ligand-receptor complexes at finite temperature. For an accurate calculation of the Gibbs free energies of binding, it is necessary to include all thermally accessible molecular conformations by Boltzmann weighting of the conformerrotamer ensemble (CRE). Conformers and rotamers are discriminated by a combination of structural (RMSD and rotational constants) and energetic threshold criteria $(\Delta \mathrm{E})$.

Hybrid and double-hybrid DFT calculation were used to verify the ligand poses and their binding energies since those methods showed a low mean absolute deviation for the S22 benchmark set of non-covalent interactions [31].

This combinatorial workflow of an exhaustive conformational and rotational sampling from meta-dynamics with different bias potentials and normal MD sampling for low energy conformers gives a representative set of CREs. The GFN2 calculated Gibbs free energies of binding for the SAMPL8 'drugs of abuse' compounds to the cucurbit[8]uril, CB[8] receptor are in excellent agreement with the recently released experimental data [32]. This demonstrates the efficiency of the CREST iterative meta-dynamics with genetic crossing (iMTD-GC) exploration of chemical space plus the accurate description of non-covalent free energies of ligand-receptor binding; it holds great promise for calculating absolute binding energies of small drug molecules in receptor binding sites.

\section{Results and Discussion}

The receptor cucurbit[8]uril (CB[8]) has a cage-like form (Figure 1) and can form complexes with different guest molecules. Ten complex ligands (Figure 2, G0 to G9) are included in this work. For ligand G0, a $\mathrm{CB}[8]^{*} \mathrm{G} 0$ 1:1 co-crystal structure is available together with experimental Gibbs free energies of binding [20]. This ligand-receptor complex thus serves as a benchmark to ensure that the GFN2 calculations are able to reproduce experimental structural parameters and CREST is able to recover experimental ligand binding free energies.

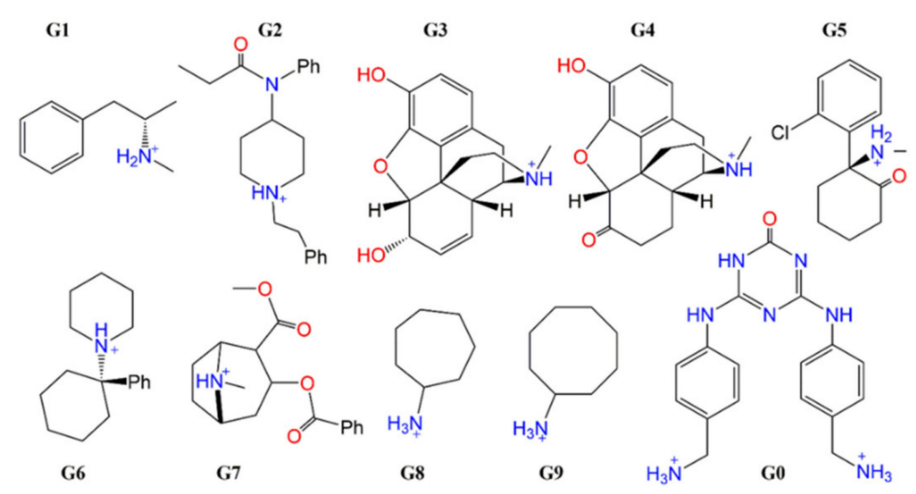

Figure 2. Structures of the guest molecules (G0-G9) used for calculations of Gibbs free energies of binding to the host $\mathrm{CB}[8]$.

\subsection{Structural Parameters of Host and Host-Guest Complexes}

In the ligand-receptor complex $\mathrm{CB}[8]^{*} \mathrm{G} 0$, the receptor has a centrosymmetric arrangement whereas the diamine ligand slightly deviates from symmetric binding. In addition, 
the diamine is disordered inside the $\mathrm{CB}[8]$ crystal structure in a 2:1 ration. Co-crystallized iodine $\left(\mathrm{I}_{2}\right)$ and most of the water molecules do not occupy symmetry-related positions. In the workflow of calculating accurate Gibbs free energies of binding, the generation of reliable poses is the first step. Figure 3 shows a comparison of the GFN2-xTB optimized structure of $\mathrm{CB}[8]$ with the crystal structure. The root mean square deviation (RMSD) of the xTB-optimized structure and the experimental one is $0.3 \AA$. This shows (i) the accuracy of the chosen computational method, and (ii) the fact that the $\mathrm{CB}[8]$ ring does not deform upon ligand binding.
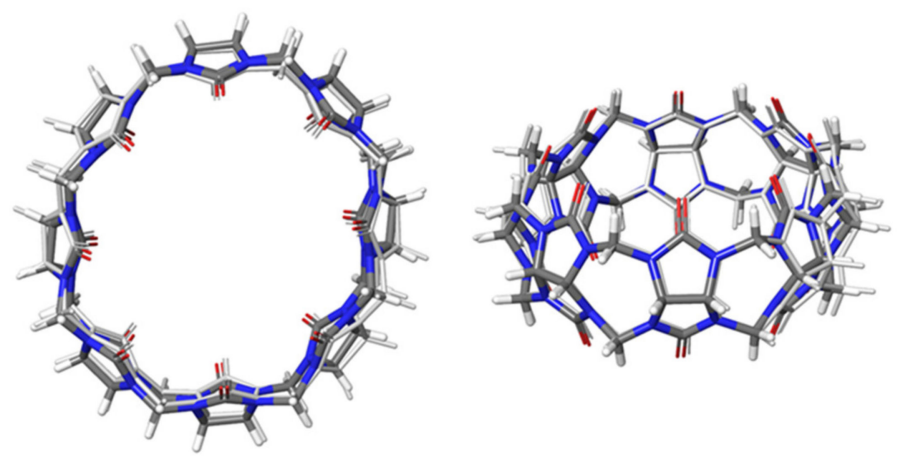

Figure 3. Structural superposition of the experimental X-ray structure of the $\mathrm{CB}[8]$ receptor (in color) and the GFN2-xTB-optimized structure. Left: Top view; Right: Side view.

The crystal structure of $\mathrm{CB}[8]$ in complex with the G0 ligand in a 1:1 stoichiometry [20] was first energy minimized. The RMSD between the $\mathrm{CB}[8]^{*} \mathrm{G} 0$ host-guest co-crystal and the GFN2-xTB optimized structures was $0.50 \AA$. In particular, the re-adjustment of the orientation of the protonated amines to form two hydrogen bonds with the carbonyl oxygen atoms led to a RMSD of $0.36 \AA$ for the G0 ligand only (see below).

We carefully assessed the degree of flexibility of the $\mathrm{CB}[8]$ receptor and its effect on the sampling of the Gibbs free energy. CREST searches of the receptor structure with and without constraining the host atoms gave identical results. Six iterative MTD runs with different bias potentials, a re-optimization of the generated conformers plus a re-ranking according to calculated Gibbs energies gave only two representative CB[8] conformers, in the presence and absence of positional constraints. The lowest conformer has a population of $99.96 \%$ and corresponds to the crystallized receptor structure. The Gibbs free energy difference between a constrained and flexible host molecule was $-0.5 \mathrm{kcal} / \mathrm{mol}$. Thus, the $\mathrm{CB}[8]$ receptor can be constrained as one entity using a force constant of 0.5 without any loss in accuracy.

Then, ligand and receptor-ligand complex were subjected to individual exhaustive CREST searches. The G0 ligand adopts a U-shape conformation inside CB[8]. A comparison of the co-crystallized ligand G0 inside the $\mathrm{CB}[8]$ receptor and the best-ranked pose according to its Gibbs free energy of the receptor-ligand complex is given in Figure 4. The small difference in Gibbs free energy of binding of G0 to the CB[8] host is $0.6 \mathrm{kcal} / \mathrm{mol}$ and shows that constraining the host molecule during CREST ligand-receptor sampling is possible without a loss in accuracy.

\subsection{The Protonation State of $G 0$}

The melamine-derived ligand G0 (1,3-bis(4-aminomethylphenyl)triazene) only slightly deforms the host in the $\mathrm{CB}[8]^{*} \mathrm{G} 0$ complex (see above). The free ligand G0 shows 12 different conformers and rotamers but complexation induces a folding process resulting in the U-shaped structure of G0 in the $\mathrm{CB}[8]^{*} \mathrm{G} 0$ complex with 8 members in the CRE. The X-ray crystal structure of host-guest complex demonstrated the excellent fit of the U-shaped conformer and the cavity of $\mathrm{CB}[8][20]$. The $\mathrm{CB}[8]^{*} \mathrm{G} 0$ complex exhibits an unusually slow dissociation with a half-life of $\sim 1$ day, which was attributed to a large conformation 
re-arrangement of the ligand before dissociation. The room temperature ${ }^{1} \mathrm{H}-\mathrm{NMR}$ spectrum in $\mathrm{D}_{2} \mathrm{O}$ was used to assign a doubly protonated states to the 4-aminomethyphenyl groups. When calculating the ligand binding free energy of the doubly protonated ligand $\mathrm{G}^{2+}$ to $\mathrm{CB}[8]$, a value of $-35.93 \mathrm{kcal} / \mathrm{mol}$ was obtained for the top-ranked pose and $-37.30 \mathrm{kcal} / \mathrm{mol}$ for the Gibbs free energy of binding for the ensemble. These values are more than a factor of two larger than experiment $(-14.67 \mathrm{kcal} / \mathrm{mol})$ and larger than that observed for all other host-guest complexes (see below). DFT single point calculations for the top-ranked binding pose gave consistent binding energies of $-40.19 \mathrm{kcal} / \mathrm{mol}$ (PWPB95/def2-TZVP) and $-45.67 \mathrm{kcal} / \mathrm{mol}$ (B2PLYP/def2-TZVP), which are in good agreement with the GFN2-xTB results but also significantly larger than the experiment.
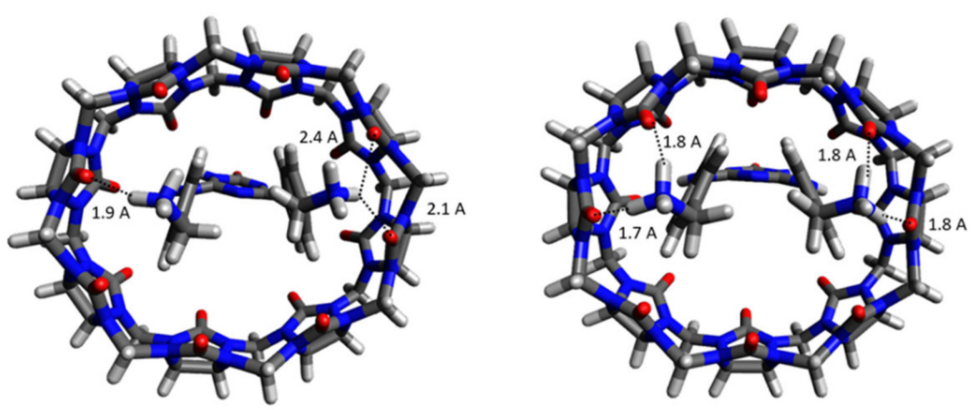

Figure 4. Ligand G0 inside the $\mathrm{CB}[8]$ receptor. Left: X-ray structure of co-crystallized complex. Right: Top-ranked ligand-receptor complex from conformational searches and ranking according to Gibbs free energies.

We then investigated the binding of a neutral $\mathrm{G}^{0}$ to $\mathrm{CB}[8]$ for which a Gibbs free energy of binding of $-4.06 \mathrm{kcal} / \mathrm{mol}$ was obtained, which is too low and not in agreement with the experiment. Only for the singly protonated $\mathrm{G0}^{+}$, reasonable ligand binding free energies of $-18.25 \mathrm{kcal} / \mathrm{mol}$ (top-ranked pose) and $-20.02 \mathrm{kcal} / \mathrm{mol}$ (ensemble) were obtained. The energies of association $\left(\Delta \mathrm{E}_{\mathrm{a}}\right)$ for the best-ranked binding mode was $-38.61 \mathrm{kcal} / \mathrm{mol}$ for GFN2-XTB; DFT calculations with PBE0 $(-31.12 \mathrm{kcal} / \mathrm{mol})$, B2PLYP $(-37.68 \mathrm{kcal} / \mathrm{mol})$ and PWPB95 $(-33.77 \mathrm{kcal} / \mathrm{mol})$ give a very consistent view and support the assignment to a mono-protonated state of $\mathbf{G 0}$. Thus, we can assign a singly protonated form to G0.

The assignment of a doubly protonated form to $\mathbf{G} 0$ was based on the interpretation of ligand ${ }^{1} \mathrm{H}-\mathrm{NMR}$ spectra [20]. In $\mathrm{D}_{2} \mathrm{O}$, the amine protons are exchanging to deuterons and not detectable in the spectrum. The equivalence of chemical shifts of the aromatic hydrogen atoms of the phenyl rings was used to suggest identical protonation states of the 4-aminomethyl groups. However, a fast proton exchange at room temperature also renders them apparently equivalent. The experimental and calculated free energies of binding are very close to those of the other ligands in this competition and allows to assign a mono-protonated state.

\subsection{Gibbs Free Energies of Ligand-Receptor Binding}

Accurately calculating the Gibbs free energies of ligand-receptor binding requires an exhaustive conformational search plus a reliable energy evaluation and Boltzmann weighting.

Table 1 gives the total number of unique structure in the CREs after conformational sampling, elimination of duplicate entries using structural and energetic criteria, followed by a re-optimization with tight convergence criteria and prior to thermodynamic corrections from Hessian calculations. The numbers of unique structures are thus corresponding to the number of free energy calculations, which need to be performed to obtain free energies of binding. It is evident that an accurate but also very efficient method is required to calculate Gibbs free energies of $136 \mathrm{CB}[8]^{*} \mathrm{G} 2$ receptor-ligand complexes, for example. 
Table 1. Number of unique structures in the conformer/rotamer ensembles (CREs) of free ligands G0-G9 and ligand-receptor complexes $\mathrm{CB}[8]^{*}$ ligand prior to final re-ranking according to Gibbs free energies.

\begin{tabular}{ccc}
\hline & $\begin{array}{c}\text { Number of Unique Entries } \\
\text { in CRE of Free Ligand }\end{array}$ & $\begin{array}{c}\text { Number of Unique Entries in the CRE } \\
\text { of the CB[8]*Ligand Complex }\end{array}$ \\
\hline G0 & 9 & 12 \\
G1 & 11 & 21 \\
G2 & 158 & 137 \\
G3 & 5 & 4 \\
G4 & 4 & 8 \\
G5 & 9 & 10 \\
G6 & 14 & 19 \\
G7 & 16 & 39 \\
G8 & 17 & 90 \\
G9 & 22 & 175 \\
\hline
\end{tabular}

The number of unique entries after the first two stages can be found in the Supplementary Information Table S2.

\subsection{Free Energies of Binding of Cyclic Anamines to CB[8]}

The binding of ligands G8 (cycloheptanamine) and G9 (cyclooctanamine) to CB[8] is a benchmark for the sufficiency of our conformational sampling, and the accuracy of ensemble-averaged Gibbs free energies of interactions (see Figure 2). As part of SAMPL6 [14] and later available experimental ligand binding affinities, they serve as competitive inhibitors for ligand binding in NMR titration experiments. As a result of CREST sampling, CREs with 90 and 175 unique entries for the ligand-receptor complexes, and 17 and 22 unique free ligand structures were generated after sequential refinement (see Table 1). This demonstrates that, albeit a low degree of free ligand conformational flexibility, the receptor-ligand complexes display a very complex potential energy surface and a multitude of distinct binding events.

Figure 5 displays an overlay of the unique entries in the ligand-receptor CREs. Due to the symmetry of the host, the G8 and G9 ligands can access the CB[8] receptor from above and below the cage. Both binding modes are fully recovered during the sampling. In the lower part of Figure 5, the top-ranked 10 binding poses of the ensembles are shown. This gives an indication of the extent of variability of ligand binding modes to the receptor in a narrow energy window of $6 \mathrm{kcal} / \mathrm{mol}$. The alkyl ring of the ligands is mainly pointing towards the inner side of the receptor where weak hydrophobic interactions are dominating. The positively charged amine groups, on the other hand, are mostly directed towards the carbonyl groups of the $\mathrm{CB}[8]$ receptor to form hydrogen bonds.

The calculated free energies of binding for the G8 and G9 ligands are -8.30 and $-8.64 \mathrm{kcal} / \mathrm{mol}$ when considering the top-ranked conformer only, compared to an ensemble $\Delta \mathrm{G}_{\text {bind,ens }}$ of -7.90 and $-8.13 \mathrm{kcal} / \mathrm{mol}$, respectively (Table 2 ). From a large number of poses, ligand-receptor complexes with 90 and 175 unique conformer entries can efficiently be generated with our approach, sampled, and ranked. The incorporation of conformers above the global minimum affects the calculated ligand binding free energies to a small degree only. In absence of any structural information, the conformational sampling plus sequential refinement steps and final re-ranking according to their Gibbs free energies is able to generate ligand binding modes in excellent agreement with experiment: for G8 and G9, the experimental binding energies of -9.18 and $-10.2 \mathrm{kcal} / \mathrm{mol}$ are only $0.8-1.6 \mathrm{kcal} / \mathrm{mol}$ lower than the GFN2-xTB results. 
$\mathrm{CB}[8]^{\star} \mathrm{G} 8$
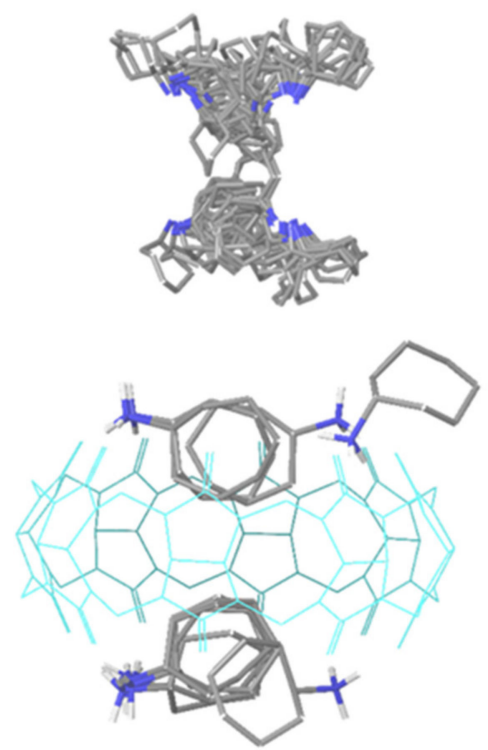

$\mathrm{CB}[8]^{\star} \mathrm{G} 9$
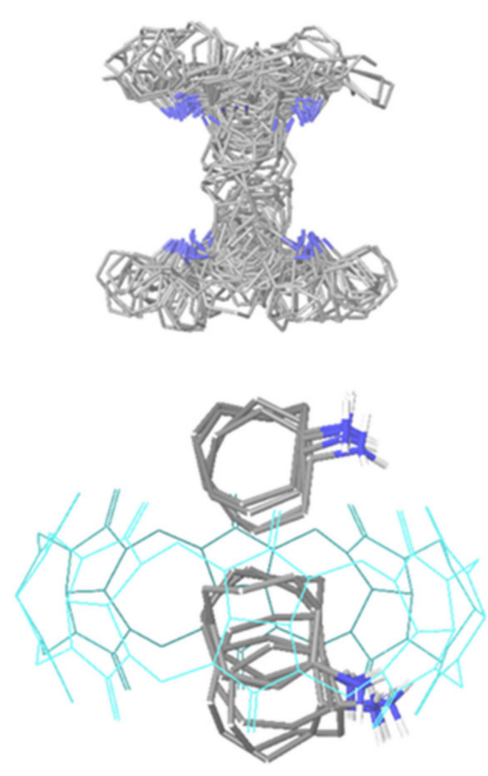

Figure 5. Top: Display of the 90 and 175 unique representatives of the CREs of the CB[8]*G8 and G9 complexes, respectively. The $\mathrm{CB}[8]$ receptor is not displayed. Bottom: Binding modes of the 10 top-ranked poses $(\mathrm{CB}[8]$ is displayed in cyan and wireframe for the sake of clarity).

Table 2. Comparison of calculated GFN2-xTB Gibbs free energies of binding of ligands G0-G9 (in $\mathrm{kcal} / \mathrm{mol}$ ) to the $\mathrm{CB}[8]$ receptor with experiment from ref [33].

\begin{tabular}{cccc}
\hline Ligand & Experiment & $\Delta \mathrm{G}_{\text {bind }}$ & $\Delta \mathrm{G}_{\text {bind,ens }}$ \\
\hline G0 & $-14.67 \pm 0.1^{\mathrm{a}}$ & -18.25 & -18.25 \\
G1 & $-7.05 \pm 0.1$ & -10.61 & -12.22 \\
G2 & $-9.94 \pm 0.06$ & -9.84 & -11.14 \\
G3 & $-11.6 \pm 0.04$ & -14.80 & -16.31 \\
G4 & $-11.2 \pm 0.1$ & -14.74 & -16.13 \\
G5 & $-12.3 \pm 0.16$ & -11.62 & -12.95 \\
G6 & $-14.1 \pm 0.04$ & -10.03 & -11.30 \\
G7 & $-7.93 \pm 0.15$ & -12.38 & -13.54 \\
G8 & $-9.18 \pm 0.03$ & -8.30 & -7.90 \\
G9 & $-10.2 \pm 0.04$ & -8.64 & -8.13 \\
\hline
\end{tabular}

${ }^{a}$ from ref. [20]; Experimental uncertainties are given. $\Delta \mathrm{G}_{\text {bind }}$ is the free energy of binding of the top-ranked pose. $\Delta G_{\text {bind,ens }}$ is the Boltzmann-weighted Gibbs free energy of binding of all CREs from Table 1.

Figure 6 shows a comparison of the GFN2-xTB G0-G9 calculated ligand binding free energies with experimental values, which were released after the SAMPL challenge. The calculated Gibbs free energies of binding (see Table 2) are in good agreement with the experiment. For one-half of the complexes, the GFN2-xTB free energies of binding are more negative than experiment. An overbinding by 3.2 and $3.5 \mathrm{kcal} / \mathrm{mol}$ is observed for ligands G3 (morphine) and G4 (hydromorphone) and by $4.5 \mathrm{kcal} / \mathrm{mol}$ for G7 (cocaine). The only structural motif that they have in common is the tricyclo-bridged hydrocarbon with a positively charged tertiary amine incorporated in one of the rings. This appears to be a critical structural issue for the xTB calculations. 


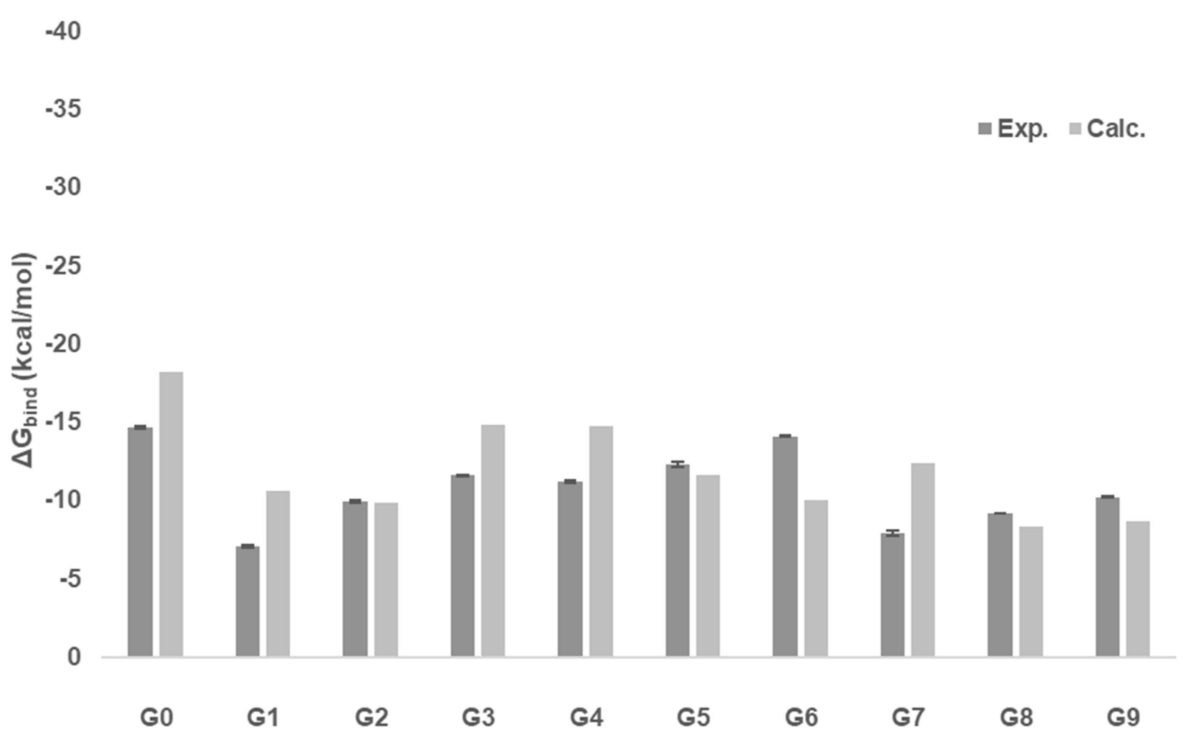

Figure 6. Calculated Gibbs free energies of binding of top-ranked ligands G0-G9 (in $\mathrm{kcal} / \mathrm{mol}$ ) to the $\mathrm{CB}[8]$ receptor in comparison to experiment.

Figure 7 displays the top-ranked poses of ligands G0 to G9 binding to the curcurbit[8] uril receptor. For methamphetamine (G1) binding to $\mathrm{CB}$ [8], free energies of binding of $-10.61 \mathrm{kcal} / \mathrm{mol}$ and $-12.22 \mathrm{kcal} / \mathrm{mol}$ were calculated for the top-ranked binding mode and the ensemble, respectively. With respect to the experimental value of $-7.05 \mathrm{kcal} / \mathrm{mol}$, the typical overbinding can be observed. The molecule is small and there are 21 unique entries in the CRE of the ligand-receptor complex (see Table 1). The phenyl ring is incorporated into the hydrophobic cavity of the host molecule. The propane-2-amine is pointing towards the solvent and the amine group forms two hydrogen bonds with carbonyl groups of $\mathrm{CB}[8]$ receptor with distances of $1.8 \AA$ and $1.9 \AA$.

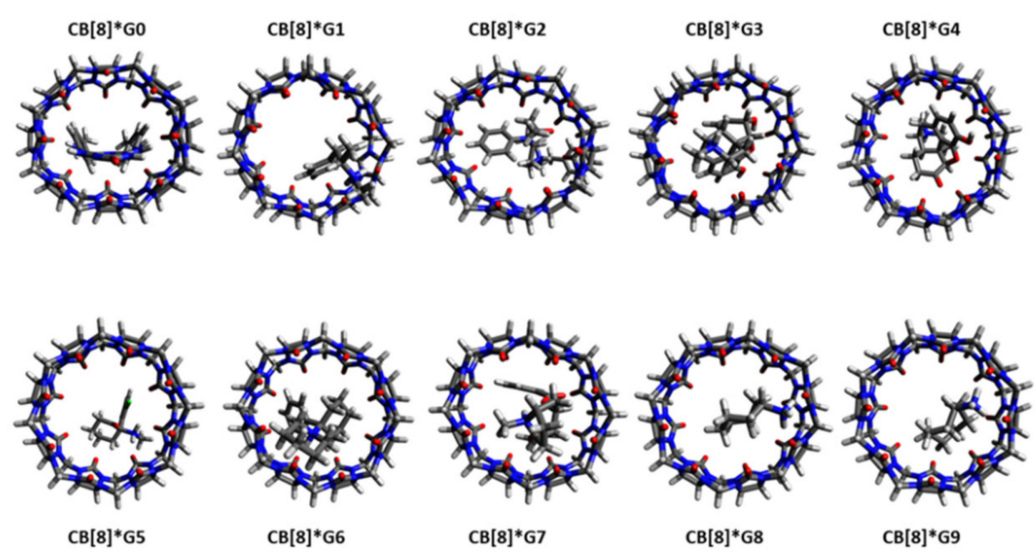

Figure 7. Structures of the top-ranked $\mathrm{CB}[8]^{*} \mathrm{G} 0-\mathrm{G} 9$ receptor-ligand complexes.

Fentanyl (G2) has a calculated free energy of binding of $-9.84 \mathrm{kcal} / \mathrm{mol}(-11.14 \mathrm{kcal} / \mathrm{mol}$ for the CRE ensemble structures). This is in excellent agreement with the experimental value of $-9.94 \mathrm{kcal} / \mathrm{mol}$. Given its large number of rotatable bonds (6), the free ligand has 158 unique conformers. Due to its elongated shape, 137 unique structures were also found for the ligand-receptor complex. The two terminal phenyl rings at each end of the complex, plus the central piperidine make this compound amphiphilic in character. The hydrophobic $\mathrm{N}$-phenyl propylamide occupies a position inside the $\mathrm{CB}[8]$ ring, the piperidine forms 
hydrogen bonds with the ring atoms at $1.8 \AA$ distance, and the (hydrophobic) ethylphenyl moiety is solvent-exposed.

The binding of morphine (G3) to the host has an experimental Gibbs free energy of $-11.6 \mathrm{kcal} / \mathrm{mol}$ which agrees well with the calculated values of $-14.80 \mathrm{kcal} / \mathrm{mol}$ and $-16.31 \mathrm{kcal} / \mathrm{mol}$. The morphine molecule is rigid and has no freely rotatable bonds. This explains why only four unique structures of morphine poses of binding in the complex are found. Whereas the 3-methyl-hexahydro isoquinoline is solvent-exposed and does not form direct interactions with the host, one diol forms a weak H-bond of $2.1 \AA$ distance with a carbonyl oxygen atom of the receptor. There are no further directed intermolecular interactions.

The experimental hydromorphine (G4) binding free energy $(-11.2 \mathrm{kcal} / \mathrm{mol})$ is close to the calculated $(-14.74$ and $-16.13 \mathrm{kcal} / \mathrm{mol})$. The $\mathrm{xTB}$ results may even reproduce the relative binding affinity differences between G4 and G3. In addition, the ligand-receptor interactions are similar. There is only one $\mathrm{H}$-bond between ligand and receptor at a distance of $2.1 \AA$.

The ligands G5 (ketamine) and G6 (phencyclidine) are similar in shape and possess 10 and 19 unique structures, respectively, in the final CRE. Ligand G5 (ketamine) does not fully insert into the $\mathrm{CB}[8]$ cavity. The top pose is positioned above the receptor: The phenyl ring is in an apical position with the chloride pointing towards the receptor's cage. The cyclohexanone ring occupies an equatorial position. Ketamine only forms one strong hydrogen bond between the amine and the carbonyl group of the receptor (distance $1.7 \AA$ ). Given the excellent agreement between calculated and experimental ligand binding free energies, we are confident to assign a +1 total charge to the system although experiments were performed at close to neutral $\mathrm{pH}$. The best-ranked phenylcyclidine ligand (G6) is, in contrast, buried in the receptor with both cylcohexane and phenyl rings inserted. The positively charged piperidine is in an apical position and pointing towards the solvent. It forms a hydrogen bond at $1.8 \AA$ distance with the receptor. The calculated free energies of binding $(-11.62 \mathrm{kcal} / \mathrm{mol}$ for top pose and $-12.95 \mathrm{kcal} / \mathrm{mol}$ for the ensemble of G5) vs. $-10.03 \mathrm{kcal} / \mathrm{mol}$ and $-11.30 \mathrm{kcal} / \mathrm{mol}$ for $\mathbf{G 6}$ are in good agreement with the respective experimental values of -12.3 and $-14.1 \mathrm{kcal} / \mathrm{mol}$.

In the receptor-ligand complex CRE of cocaine (ligand G7) 39 unique structures were found. The deviation between experiment $(-7.93 \mathrm{kcal} / \mathrm{mol})$ and XTB calculations $(-12.38 \mathrm{kcal} / \mathrm{mol}$ for top pose and $-13.54 \mathrm{kcal} / \mathrm{mol}$ for the ensemble population $)$ is $4.5-$ $5.5 \mathrm{kcal} / \mathrm{mol}$ and thus the largest of the set. The azabicyclooctane is located above the receptor; the benzoyloxy and methyl carboxylate groups are fully incorporated into the receptor. There is no directed interaction between ligand and receptor.

Figure 8 gives the deviation of $\mathrm{xTB}$ calculated Gibbs free energies of binding from experiment (see Table 2). A positive value refers to an overbinding of GFN2-xTB calculations. In 7 out of the 10 complexes, this overbinding is apparent. When only the top pose is considered, the mean absolute error (MAE) is $2.6 \mathrm{kcal} / \mathrm{mol}$, which increases to $3.38 \mathrm{kcal} / \mathrm{mol}$ when including the Boltzmann-weighted ensemble population (see Table 3). When the change in conformational and rotational entropy is considered $\left(-\mathrm{T} \Delta \mathrm{S}_{\mathrm{CR}}\right)$, the error is $3.43 \mathrm{kcal} / \mathrm{mol}$.

Table 3. Error of GFN2-xTB CB[8]*(G0-G9) calculated ligand binding free energies in kcal/mol.

\begin{tabular}{cccc}
\hline & $\Delta \mathbf{G}_{\text {top-pose }}$ & $\Delta \mathbf{G}_{\text {ensemble }}$ & $\Delta \mathbf{G}_{\text {ensemble }}-\mathbf{T} \boldsymbol{\Delta} \mathbf{S}_{\mathbf{C R}}$ \\
\hline RMSE & 2.97 & 3.86 & 3.77 \\
MAE & 2.56 & 3.38 & 3.43 \\
SD & 1.4 & 2.0 & 1.6 \\
\hline
\end{tabular}




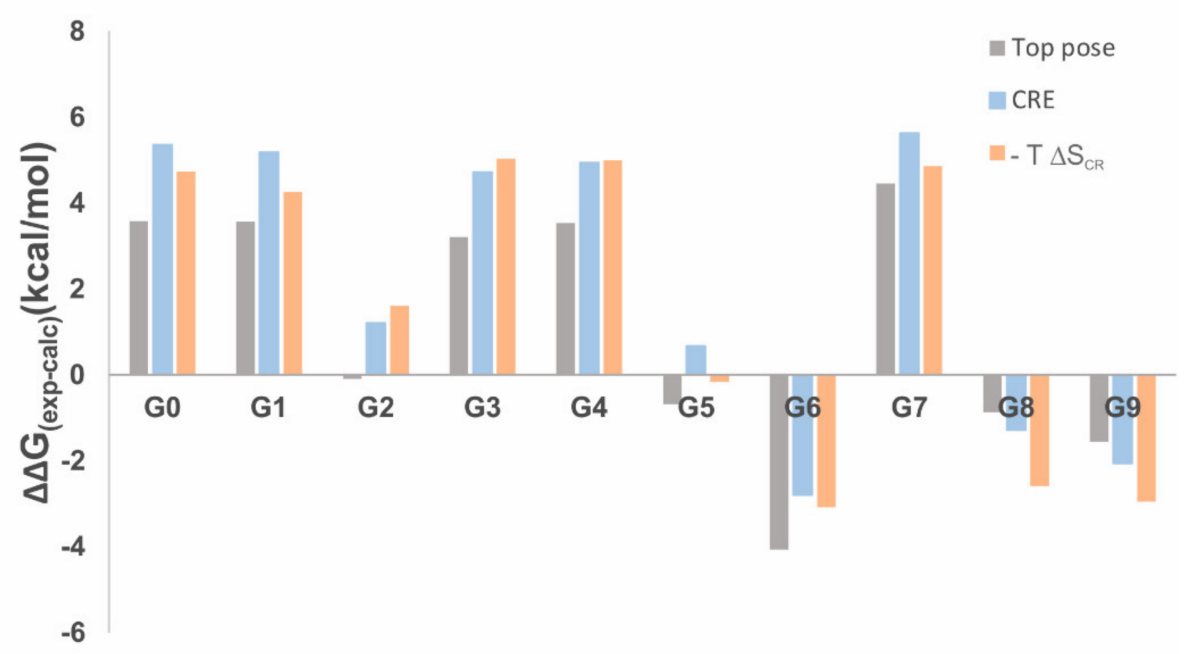

Figure 8. Deviations of GFN2-xTB CREST results from experiment in kcal/mol for ligands G0 to G9 binding to $\mathrm{CB}[8]$. Grey: Gibbs free energy of binding for top pose only; blue: For all members of the CRE; orange: Taking into account changes in conformational and rotational entropy of the ensemble $\left(-\mathrm{T} \Delta \mathrm{S}_{\mathrm{CR}}\right)$.

\subsection{Comparison of GFN2-xTB Results with DFT}

The GFN2-xTB Hamiltonian is especially parametrized for properties such as geometries, vibrational frequencies, and non-covalent interactions [34]. Usually, covalent bond energies are systematically overestimated by this method. For non-covalent interactions, however, GFN2-xTB outperforms GFN-xTB and PBE0-D3(BJ) without specific terms in the Hamiltonian to treat hydrogen bonds.

Table 4 compares the energies of association $\left(\mathrm{E}_{\mathrm{a}}\right)$ from GFN2-xTB with DFT calculations using hybrid PBE0, double-hybrid B2PLYP and the spin-opposite-scaled double hybrid functional PWPB95 of ligands G0-G9 with the CB[8] receptor. GFN2-xTB shows the abovementioned systematic overbinding and the calculated energies of association are larger by up to $25 \mathrm{kcal} / \mathrm{mol}$ relative to DFT calculations.

Table 4. Energies of association ( $\mathrm{E}_{\mathrm{a}}$ ) of GFN2-xTB and DFT calculations for top-ranked $\mathrm{CB}[8]^{*} \mathrm{G} 0-\mathrm{G} 1$ ligand-receptor complexes in $\mathrm{kcal} / \mathrm{mol}$.

\begin{tabular}{ccccc}
\hline $\begin{array}{c}\text { CB[8] } \\
\text { Ligand }\end{array}$ & GFN2-xTB & PBE0 & B2PLYP & PWPB95 \\
\hline G0 & -38.61 & -31.12 & -37.68 & -33.77 \\
G1 & -28.39 & -19.57 & -21.68 & -19.66 \\
G2 & -30.34 & -18.63 & -23.20 & -20.30 \\
G3 & -35.28 & -22.31 & -27.55 & -24.42 \\
G4 & -35.79 & -19.12 & -24.51 & -21.44 \\
G5 & -31.26 & -22.57 & -28.41 & -25.15 \\
G6 & -28.31 & -25.57 & -29.70 & -28.59 \\
G7 & -28.69 & -11.53 & -15.94 & -12.96 \\
G8 & -22.46 & -20.86 & -21.69 & -19.87 \\
G9 & -24.51 & -22.37 & -23.54 & -21.44 \\
\hline
\end{tabular}

All DFT results give a very consistent picture of the energies of association of ligands G0-G9 with the receptor. Hybrid, double-hybrid, and spin-opposite-scaled double hybrid functionals agree to within $4-7 \mathrm{kcal} / \mathrm{mol}$. This is a strong indicator of the reliability and accuracy of the GFN2 generated best binding poses. The generated binding modes are very well suited for any subsequent higher-level calculation.

The DFT energies of association are consistently lower than the GFN2-xTB results. B2PLYP calculations give larger $\mathrm{E}_{\mathrm{a}}$ (by a mean of $-4 \mathrm{kcal} / \mathrm{mol}$ ) than PBE0; and PWPB95 
(with a mean deviation of $-1.4 \mathrm{kcal} / \mathrm{mol}$ from PBE0 results) is in between. However, since GFN2 is particularly parametrized for the thermochemistry of non-covalent interactions, a comparison between the GFN2 Gibbs free energies and DFT electronic energies appears more plausible.

\section{Materials and Methods}

\subsection{Host-Guest Starting Structures}

The initial structures of the individual host and guest molecules (Figure 2) were taken from the from the SAMPL8 repository (https: / / zenodo.org/record/4029560; accessed on 13 March 2021) [35]. The host molecule cucurbit[8] uril (CB[8]) is a cyclic neutral structure with eight carboxylic acid groups. Its cage-like form enables the formation of non-covalent receptor-ligand complexes with a diverse set of ligands. This cavity has a hydrophobic binding pocket, which eases the binding upon weak hydrophobic interactions. We took the $\mathrm{CB}[8]$ crystal structure from ref [20] as the receptor structure.

After carefully checking the effect of the host flexibility, we constrain the CB[8] during the simulations.

Due to the $\mathrm{pK}_{\mathrm{a}}$ value of the ligands' amine groups and the given experimental conditions of SAMPL8 challenge $\left(\mathrm{pH}=7.4\right.$ at $\left.25^{\circ} \mathrm{C}\right)$, all the ligands were submitted to the calculations in their protonated states. The ligands G1-G9 (Figure 2) are +1 charged, whereas the co-crystallized G0 ligand has a charge of +2 [20]. A GBSA solvent model for water used and there were no counterions present. Experimental conditions were as follows: The buffer was $20 \mathrm{mM}$ sodium phosphate buffer at $\mathrm{pH}$ 7.4. Guest concentrations were in the 0.5 to $1.5 \mathrm{mM}$ range and CB8 concentrations between 0.025 to $0.1 \mathrm{mM}$. All binding stoichiometries have been validated by repeat ITC experiments and by NMR spectroscopy binding studies. Ligands G0-G9 were created with Maestro and stored as Cartesian coordinations.

They were manually positioned inside the $\mathrm{CB}[8]$ receptor structure. These are the co-crystallized ligand 1,3-bis(4-aminomethylphenly)triazene (G0), methamphetamine hydrochloride (G1), fentanyl citrate (G2), morphine hydrochloride (G3), hydromorphine hydrochloride (G4), ketamine hydrochloride (G5), phencyclidine hydrochloride (G6), cocaine hydrochloride (G7), cycloheptanamine hydrochloride (G8), and cyclooctanamine hydrochloride (G9). We also invested the possibility of chiral discrimination by the receptor; for example, the difference in Gibbs free energy of binding of R- and S-enantiomers of G5 to $\mathrm{CB}[8]$ was below $1 \mathrm{kcal} / \mathrm{mol}$ and not further considered.

\subsection{Structural Optimization and Free Energies of Binding}

For the geometry optimizations and sampling of the host-ligand complexes, we have used CREST [30,36] (Conformer-Rotamer Ensemble Sampling Tool, v2.10.2; available from https:/ / github.com/grimme-lab/crest, accessed on 13 March 2021) and GFN2-xTB (v6.3.2; available from https:/ / github.com/grimme-lab/xtb, accessed on 13 March 2021) [37,38].

Due to the large number of atoms in biologically relevant structures and the flexibility of either receptor and/or ligands, full QM calculations are time-consuming and impractical for most application to these systems. XTB is able to perform very fast and accurate semiempirical quantum mechanical calculations [39] including geometry optimizations for complexes of 200-300 atoms, vibrational frequencies, and thermodynamic corrections in the rigid-rotor harmonic oscillator (RRHO) approximation [34]. The Generalized Born (GB) model augmented with the hydrophobic solvent accessible surface area (SA) term (GBSA) was used to model solvent effects.

Initial binding poses of the host-guest systems were generated manually by positioning the ligand close to the center of the receptor. Then, the geometries were refined in order to remove steric clashes. 
The free energy of binding of the top-ranked pose is calculated at $\mathrm{T}=298.15 \mathrm{~K}$ in aqueous solution according to Equation (1):

$$
\Delta G_{\text {binding }}=\Delta E_{(\text {complex-receptor-ligand })}+\Delta G_{R R H O}^{T}+\Delta G_{G B S A}
$$

The Gibbs free energy of the ensemble $\Delta \mathrm{G}_{\text {bind,ens }}$ is the Boltzmann-weighted sum of all entries within an energy window of $6 \mathrm{kcal} / \mathrm{mol}$ above the global minimum. The conformational entropy of an ensemble is the entropy associated with the number of conformations of the molecule in the energy window. To calculate the conformational entropy, the possible conformations of the molecule are discretized into a finite number of states according to the CRE criteria. It is then calculated as the probability-weighted occupancy of each structure in the ensemble as $S_{C R}=-R \Sigma_{i} p_{i} \log p_{i}$, where $R$ is the gas constant and $p_{i}$ is the probability of the molecule to be in state ${ }_{i}$.

The ensemble entropy is also linked to the ensemble free energy $\mathrm{G}_{\mathrm{CR}}=-\mathrm{T} \cdot \mathrm{S}_{\mathrm{CR}}$. It is common for a free ligand to lose conformational entropy upon forming a ligand-receptor complex. Here, sometimes the ligand in the receptor shows more possible binding modes than the free ligand has accessible conformers in solution.

\subsection{The Conformer/Rotamer Ensemble}

The exploitation of the conformational space by conventional sampling methods is limited. Here, meta-dynamic molecular dynamics simulations (MTD) are a superior tool for an extensive sampling of states and extraction of individual conformers. It establishes more reliable ensembles at a good accuracy/computational cost ratio [40]. The iMTDGC procedure in CREST is a combined approach of extensive meta-dynamic sampling (MTD), with a final additional genetic Z-matrix crossing (GC) step in order to give a final conformer/rotamer ensemble (CRE). For complexes of $\mathrm{CB}[8]$ and G0 to G9, the non-covalent interaction (NCI) mode creates an additional polynomial ellipsoid wall potential, which is added to the meta-dynamics. This avoids the dissociation of weakly bound aggregates.

XTB calculations are combined with root-mean-square-deviation (RMSD) based metadynamics by applying a Gaussian-type biasing potential to previous minima on the PES (Equation (2)):

$$
V_{\text {bias }}=\sum_{i}^{n} k_{i} \exp \left(-\alpha \Delta_{i}^{2}\right)
$$

where $\Delta_{\mathrm{i}}$ are collective variables, $n$ is the number of reference structures, $k_{i}$ are the pushing strengths, and $\alpha$ determines the potential shape. The MTD simulation length is determined automatically by a flexibility measure of the molecule. Several independent MTDs (at $300 \mathrm{~K}$ ) are performed with different settings for $\alpha\left(\mathrm{in} \mathrm{Bohr}^{-1}\right.$ ) and $k_{i} / N$ (in $\mathrm{mEh}$ ) (see Table S1)

The default settings are employed for the iMTD-GC procedure in CREST. The MTD simulation length is determined automatically by a flexibility measure of the molecule and varying settings for $\alpha$ and $k i / N$. Snapshots are geometry optimized in a multi-level, three-step-filtering procedure by applying two loose threshold settings. Short regular MD simulations are performed on the 6 lowest conformers at 400 and $500 \mathrm{~K}$ and followed by very tightly converged optimization and energy windows of 15,10 , and $6 \mathrm{kcal} / \mathrm{mol}$.

The resulting ensembles were further refined and re-ranked using tighter optimization criteria. All structures were filtered with respect to their root-mean-square-deviation (RMSD), rotational constant (Be) and relative energies within $6.00 \mathrm{kcal} / \mathrm{mol}$ of the global minimum. Energy and matching structural criteria were employed to discard duplicates. In the next step, six iterative MD sampling runs were performed starting from the lowest energy conformers. All unique conformers and rotamers within the $6 \mathrm{kcal} / \mathrm{mol}$ energy window were assembled into a conformer/rotamer ensemble (CRE). Entries in the CREs were used for further optimization with tighter optimization criteria to generate the final ensemble of unique structures. 
The NCI wall potential is removed for geometry optimizations. For each member of the final ensemble, structural optimizations with very tight convergence criteria and then vibrational frequencies were calculated in order to obtain thermodynamic corrections and Gibbs free energies. Finally, conformers in these ensembles were re-ranked according to their Gibbs free energies. Top-ranked poses of each ensemble were submitted further to higher level DFT calculations.

\subsection{DFT Calculations}

Hybrid DFT PBE0-D3 [41,42], double-hybrid B2PLYP [43], and double-hybrid-metaGGA PWPB95 [44] were performed using ORCA v4.2.1 [45,46] in order to benchmark the GFN2-xTB energies of association. For these single point calculations, a def2-TZVP basis set with corresponding auxiliary basis sets and the RIJCOSX approximation were employed in order to reduce the computational cost [47-49]. Weak non-covalent interactions were considered by using D3 dispersion corrections with Becke-Johnson damping [29,50]. Solvation effects were considered using a CPCM implicit solvent model for water [51]. Only for G7, a structural re-optimization at the TPSS-D3(BJ)/def2TZVP level was performed.

\section{Conclusions}

The blind prediction of accurate ligand-receptor poses and free energies of binding shows that the efficient GFN2-xTB method can be combined with conformational sampling using meta-MD and normal MD in CREST. An elimination of duplicates in terms of structural criteria (RMSD and rotational constants) and energetics yields the conformationrotamer ensemble CRE. With subsequent re-ranking steps using tighter criteria, finally the calculated free energies of binding of either the top poses only or the entire CRE are in good agreement with experiment.

The mean absolute error is comparable to that of usual predictions in the SAMPL host-guest competitions. Most of the entries, however, rely on a pre-parametrization of non-polarizable atomic partial charges from QM calculations. CREST with GFN2-xTB directly enters at the sampling stage without the need of a parametrization. It is also not relying on a priori knowledge of possible ligand binding modes.

For related $\mathrm{CB}$ [7] complexes, meta-GGA PW6B95 single point calculations with a COSMO-RS solvation model at TPSS-D3(COSMO) optimized structures gave a MAD of $2.02 \pm 0.46 \mathrm{kcal} / \mathrm{mol}$ but energies were reported relative to the experimental free binding energy of CB[7] ${ }^{*} \mathbf{1}$ [29]. Single-structure, non-dynamic models were generated by hand and selected candidate poses were subsequently optimized.

In this work, a comparable accuracy can be obtained without any manual intervention and experimental reference data. Due to the impressive computational speed and efficacy, CREST can also be applied to ligand-protein complexes. The ligand conformations in a drug-binding site can efficiently be sampled and provide reliable free energies of interactions. Such an approach will be of relevance also for covalent ligand binding, non-standard states of protonation, or post-translational modifications of either the ligand or receptor.

Supplementary Materials: The following are available online at https:/ / www.mdpi.com/1422-006 7/22/6/3078/s1.

Author Contributions: E.B.: Data curation, investigation, formal analysis, writing—original draft. M.S.: Conceptualization, funding acquisition, supervision, data curation, writing-review and editing. All authors have read and agreed to the published version of the manuscript.

Funding: This research was funded by the Max Planck Society for the Advancement of Science is gratefully acknowledged. The work was also supported by the European Union program ERDF (European Regional Development Fund) of the Ministery of Economy, Science and Digitalisation in Saxony Anhalt within the Center of Dynamic Systems (ZS/2016/04/78155). Gefördert durch die Deutsche Forschungsgemeinschaft (DFG)-TRR 63 “Integrierte chemische Prozesse in flüssigen Mehrphasensystemen" (Teilprojekt A4)-56091768. Funded by the Deutsche Forschungsgemeinschaft 
(DFG, German Research Foundation)-TRR 63 “Integrated Chemical Processes in Liquid Multiphase Systems" (subproject A4)-56091768. The APC was funded by Max Planck Digital Library (MPDL).

Institutional Review Board Statement: Not applicable.

Informed Consent Statement: Not applicable.

Data Availability Statement: The data presented in this study are openly available as Supplementary Materials.

Acknowledgments: We appreciate the National Institutes of Health for its support of the SAMPL project via R01GM124270 to David L. Mobley (UC Irvine).

Conflicts of Interest: The authors declare no conflict of interest.

\section{References}

1. Mobley, D.L.; Gilson, M.K. Predicting binding free energies: Frontiers and benchmarks. Annu. Rev. Biophys. 2017, 46, 531-558. [CrossRef]

2. Rosenbaum, M.I.; Clemmensen, L.S.; Bredt, D.S.; Bettler, B.; Strømgaard, K. Targeting receptor complexes: A new dimension in drug discovery. Nat. Rev. Drug Discov. 2020, 19, 884-901. [CrossRef]

3. Yang, S.Q.; Ye, Q.; Ding, J.J.; Yin, M.Z.; Lu, A.P.; Chen, X.; Hou, T.J.; Cao, D.S. Current advances in ligand-based target prediction. WIREs Comput. Mol. Sci. 2020, e1504. [CrossRef]

4. Rubio-Ruiz, B.; Serrán-Aguilera, L.; Hurtado-Guerrero, R.; Conejo-García, A. Recent advances in the design of choline kinase $\alpha$ inhibitors and the molecular basis of their inhibition. Med. Res. Rev. 2020, 41, 902-927. [CrossRef] [PubMed]

5. Homeyer, N.; Stoll, F.; Hillisch, A.; Gohlke, H. Binding free energy calculations for lead optimization: Assessment of their accuracy in an industrial drug design context. J. Chem. Theory Comput. 2014, 10, 3331-3344. [CrossRef] [PubMed]

6. Del Rio, A.; Hayes, J.M.; Stein, M.; Piras, P.; Roussel, C. Theoretical reassessment of Whelk-O1 as an enantioselective receptor for 1-(4-halogeno-phenyl)-1-ethylamine derivatives. Chirality 2004, 16, S1-S11. [CrossRef]

7. Hayes, J.M.; Stein, M.; Weiser, J. Accurate calculations of ligand binding free energies: Chiral separation with enantioselective receptors. J. Phys. Chem. A 2004, 108, 3572-3580. [CrossRef]

8. Ragusa, A.; Rossi, S.; Hayes, J.M.; Stein, M.; Kilburn, J.D. Novel enantioselective receptors for N-protected glutamate and aspartate. Chem. A Eur. J. 2005, 11, 5674-5688. [CrossRef] [PubMed]

9. Gilson, M.K.; Zhou, H.X. Calculation of protein-ligand binding affinities. Annu. Rev. Biophys. Biomol. Struct. 2007, 36, 21-42. [CrossRef]

10. Lamb, M.L.; Jorgensen, W.L. Computational approaches to molecular recognition. Curr. Opin. Chem. Biol. 1997, 1, 449-457. [CrossRef]

11. Jorgensen, W.L. The many roles of computation in drug discovery. Science 2004, 303, 1813-1818. [CrossRef]

12. Kollman, P.A.; Massova, I.; Reyes, C.; Kuhn, B.; Huo, S.; Chong, L.; Lee, M.; Lee, T.; Duan, Y.; Wang, W.; et al. Calculating structures and free energies of complex molecules: Combining molecular mechanics and continuum models. Acc. Chem. Res. 2000, 33, 889-897. [CrossRef] [PubMed]

13. Rizzi, A.; Jensen, T.; Slochower, D.R.; Aldeghi, M.; Gapsys, V.; Ntekoumes, D.; Bosisio, S.; Papadourakis, M.; Henriksen, N.M.; de Groot, B.L.; et al. The SAMPL6 sampling challenge: Assessing the reliability and efficiency of binding free energy calculations. J. Comput. Aided Mol. Des. 2020, 34, 601-633. [CrossRef]

14. Rizzi, A.; Murkli, S.; McNeill, J.N.; Yao, W.; Sullivan, M.; Gilson, M.K.; Chiu, M.W.; Isaacs, L.; Gibb, B.C.; Mobley, D.L.; et al. Overview of the SAMPL6 host-guest binding affinity prediction challenge. J. Comput. Aided Mol. Des. 2018, 32, 937-963. [CrossRef]

15. Yin, J.; Henriksen, N.M.; Slochower, D.R.; Shirts, M.R.; Chiu, M.W.; Mobley, D.L.; Gilson, M.K. Overview of the SAMPL5 host-guest challenge: Are we doing better? J. Comput. Aided Mol. Des. 2017, 31, 1-19. [CrossRef]

16. Muddana, H.S.; Daniel Varnado, C.; Bielawski, C.W.; Urbach, A.R.; Isaacs, L.; Geballe, M.T.; Gilson, M.K. Blind prediction of host-guest binding affinities: A new SAMPL3 challenge. J. Comput. Aided Mol. Des. 2012, 26, 475-487. [CrossRef]

17. Kim, J.; Jung, I.-S.; Kim, S.-Y.; Lee, E.; Kang, J.-K.; Sakamoto, S.; Yamaguchi, K.; Kim, K. New cucurbituril homologues: Syntheses, isolation, characterization, and X-ray crystal structures of cucurbit[n]uril (n = 5, 7, and 8). J. Am. Chem. Soc. 2000, 122, 540-541. [CrossRef]

18. Barrow, S.J.; Kasera, S.; Rowland, M.J.; del Barrio, J.; Scherman, O.A. Cucurbituril-based molecular recognition. Chem. Rev. 2015, 115, 12320-12406. [CrossRef] [PubMed]

19. Biedermann, F.; Scherman, O.A. Cucurbit[8]uril mediated donor-acceptor ternary complexes: A model system for studying charge-transfer interactions. J. Phys. Chem. B 2012, 116, 2842-2849. [CrossRef]

20. Liu, S.; Ruspic, C.; Mukhopadhyay, P.; Chakrabarti, S.; Zavalij, P.Y.; Isaacs, L. The cucurbit[n]uril family: Prime components for self-sorting systems. J. Am. Chem. Soc. 2005, 127, 15959-15967. [CrossRef]

21. Murkli, S.; McNeill, J.N.; Isaacs, L. Cucurbit 8 uril center dot guest complexes: Blinded dataset for the SAMPL6 challenge. Supramol. Chem. 2019, 31, 150-158. [CrossRef] 
22. Guan, D.; Lui, R.; Matthews, S. LogP prediction performance with the SMD solvation model and the M06 density functional family for SAMPL6 blind prediction challenge molecules. J. Comput. Aided Mol. Des. 2020, 34, 511-522. [CrossRef]

23. Sun, Z.X.; He, Q.L.; Li, X.; Zhu, Z.D. SAMPL6 host-guest binding affinities and binding poses from spherical-coordinates-biased simulations. J. Comput. Aided Mol. Des. 2020, 34, 589-600. [CrossRef]

24. Papadourakis, M.; Bosisio, S.; Michel, J. Blinded predictions of standard binding free energies: Lessons learned from the SAMPL6 challenge. J. Comput. Aided Mol. Des. 2018, 32, 1047-1058. [CrossRef] [PubMed]

25. Eken, Y.; Patel, P.; Diaz, T.; Jones, M.R.; Wilson, A.K. SAMPL6 host-guest challenge: Binding free energies via a multistep approach. J. Comput. Aided Mol. Des. 2018, 32, 1097-1115. [CrossRef] [PubMed]

26. Song, L.F.; Bansal, N.; Zheng, Z.; Merz, K.M. Detailed potential of mean force studies on host-guest systems from the SAMPL6 challenge. J. Comput. Aided Mol. Des. 2018, 32, 1013-1026. [CrossRef] [PubMed]

27. Mikulskis, P.; Genheden, S.; Rydberg, P.; Sandberg, L.; Olsen, L.; Ryde, U. Binding affinities in the SAMPL3 trypsin and host-guest blind tests estimated with the MM/PBSA and LIE methods. J. Comput. Aided Mol. Des. 2012, 26, 527-541. [CrossRef]

28. Grimme, S. Supramolecular binding thermodynamics by dispersion-corrected density functional theory. Chem. A Eur. J. 2012, 18, 9955-9964. [CrossRef]

29. Sure, R.; Antony, J.; Grimme, S. Blind prediction of binding affinities for charged supramolecular host-guest systems: Achievements and shortcomings of DFT-D3. J. Phys. Chem. B 2014, 118, 3431-3440. [CrossRef]

30. Pracht, P.; Bohle, F.; Grimme, S. Automated exploration of the low-energy chemical space with fast quantum chemical methods. Phys. Chem. Chem. Phys. 2020, 22, 7169-7192. [CrossRef]

31. Schwabe, T.; Grimme, S. Double-hybrid density functionals with long-range dispersion corrections: Higher accuracy and extended applicability. Phys. Chem. Chem. Phys. 2007, 9, 3397-3406. [CrossRef] [PubMed]

32. Murkli, S.; Klemm, J.; Brocket, A.T.; Shuster, M.; Briken, V.; Roesch, R.M.; Isaacs, L. In vitro and in vivo sequestration of phencyclidine by Me4Cucurbit [8] uril. Chem. A Eur. J. 2021, 27, 3098-3105. [CrossRef] [PubMed]

33. Isaacs, L. CB8-DOA-SAMPL-Answer-Sheet-20201014.Pdf. Available online: https://github.com/samplchallenges/SAMPL8 /blob/master/host_guest/Analysis/ExperimentalMeasurements/CB8-DOA-SAMPL-Answer-Sheet-20201014.pdf (accessed on 13 March 2021).

34. Bannwarth, C.; Ehlert, S.; Grimme, S. GFN2-xTB—-an accurate and broadly parametrized self-consistent tight-binding quantum chemical method with multipole electrostatics and density-dependent dispersion contributions. J. Chem. Theory Comput. 2019, 15, 1652-1671. [CrossRef]

35. Mobley, D.L.; Amezcua, M. The SAMPL8 CB8 “Drugs of Abuse" Challenge. Available online: https://github.com/ samplchallenges/SAMPL8/blob/master/host_guest/CB8/README.md (accessed on 21 February 2021).

36. Grimme, S.; Pracht, P. CREST Conformer-Rotamer Ensemble Sampling Tool Based on the GFN-; Mulliken Center for Theoretical Chemistry, University of Bonn: Bonn, Germany, 2020.

37. Bannwarth, C.; Caldeweyher, E.; Ehlert, S.; Hansen, A.; Pracht, P.; Seibert, J.; Spicher, S.; Grimme, S. Extended tight-binding quantum chemistry methods. WIREs Comput. Mol. Sci. 2020, 11, e1493. [CrossRef]

38. Grimme, S. Semi-empirical Extended Tight-Binding Program Package xtb.v- 6.3.2; Mulliken Center for Theoretical Chemistry, University of Bonn: Bonn, Germany, 2020.

39. Schmitz, S.; Seibert, J.; Ostermeir, K.; Hansen, A.; Göller, A.H.; Grimme, S. Quantum chemical calculation of molecular and periodic peptide and protein structures. J. Phys. Chem. B 2020, 124, 3636-3646. [CrossRef] [PubMed]

40. Grimme, S. Exploration of chemical compound, conformer, and reaction space with meta-dynamics simulations based on tight-binding quantum chemical calculations. J. Chem. Theory Comput. 2019, 15, 2847-2862. [CrossRef] [PubMed]

41. Grimme, S. Accurate description of van der Waals complexes by density functional theory including empirical corrections. $J$. Comput. Chem. 2004, 25, 1463-1473. [CrossRef]

42. Ernzerhof, M.; Scuseria, G.E. Assessment of the Perdew-Burke-Ernzerhof exchange-correlation functional. J. Chem. Phys. 1999, 110, 5029-5036. [CrossRef]

43. Grimme, S. Semiempirical hybrid density functional with perturbative second-order correlation. J. Chem. Phys. 2006, 124, 034108. [CrossRef] [PubMed]

44. Goerigk, L.; Grimme, S. Efficient and accurate double-hybrid-meta-gga density functionals-evaluation with the extended GMTKN30 database for general main group thermochemistry, kinetics, and noncovalent interactions. J. Chem. Theory Comput. 2011, 7, 291-309. [CrossRef]

45. Neese, F. The Orca program system. WIREs Comput. Mol. Sci. 2012, 2, 73-78. [CrossRef]

46. Neese, F. Software update: The Orca program system, version 4.0. WIREs Comput. Mol. Sci. 2018, 8, e1327. [CrossRef]

47. Neese, F. An improvement of the resolution of the identity approximation for the formation of the Coulomb matrix. J. Comput. Chem. 2003, 24, 1740-1747. [CrossRef] [PubMed]

48. Weigend, F.; Ahlrichs, R. Balanced basis sets of split valence, triple zeta valence and quadruple zeta valence quality for $H$ to Rn: Design and assessment of accuracy. Phys. Chem. Chem. Phys. 2005, 7, 3297-3305. [CrossRef] [PubMed]

49. Eichkorn, K.; Treutler, O.; Öhm, H.; Häser, M.; Ahlrichs, R. Auxiliary basis sets to approximate coulomb potentials. Chem. Phys. Lett. 1995, 240, 283-290. [CrossRef] 
50. Grimme, S.; Ehrlich, S.; Goerigk, L. Effect of the damping function in dispersion corrected density functional theory. J. Comput. Chem. 2011, 32, 1456-1465. [CrossRef]

51. Marenich, A.V.; Cramer, C.J.; Truhlar, D.G. Universal solvation model based on solute electron density and on a continuum model of the solvent defined by the bulk dielectric constant and atomic surface tensions. J. Phys. Chem. B 2009, 113, 6378-6396. [CrossRef] [PubMed] 